\title{
A Rate-Dependent Model for Metals Based on a Master Curve of Normalized Hardening Behavior of DP Steels
}

\author{
Juner Zhu ${ }^{1,2} \cdot$ Yong $\mathrm{Xia}^{1} \cdot$ Qing Zhou $^{1} \cdot$ Tomasz Wierzbicki $^{2}$
}

Received: 26 February 2016/ Accepted: 31 March 2016/Published online: 8 April 2016

(C) Society for Experimental Mechanics, Inc 2016

\begin{abstract}
A DP780 steel is tested under a wide range of strain rate from quasi-static to $1200 \mathrm{~s}^{-1}$. Positive strain rate sensitivity in flow strength and generally negative strain rate dependence in the plastic strain at the onset of necking are observed. A two-dimensional scaling approach is put forward for data analysis, which employs the necking point as a characteristic point to normalize the hardening curves. A master curve is observed, i.e. the normalized hardening curves almost superimpose upon each other and therefore can be regarded as independent of strain rate. Based on this observation, a new phenomenological model is proposed, the accuracy of which is validated by strain rate jump test. Moreover, four more DP steels, including one tested by the authors and three extracted from open publications, are investigated. It is found that master curve exists for almost all of these DP steels, and consequently the proposed model has good applicability for these materials.
\end{abstract}

\section{Graphical Abstract}

Yong Xia

xiayong@tsinghua.edu.cn

1 State Key Laboratory of Automotive Safety and Energy, Department of Automotive Engineering, Tsinghua University, Beijing 100084, People's Republic of China

2 Impact and Crashworthiness Lab, Massachusetts Institute of Technology, 77 Massachusetts Ave, Room 5-218A, Cambridge, MA 02139, USA
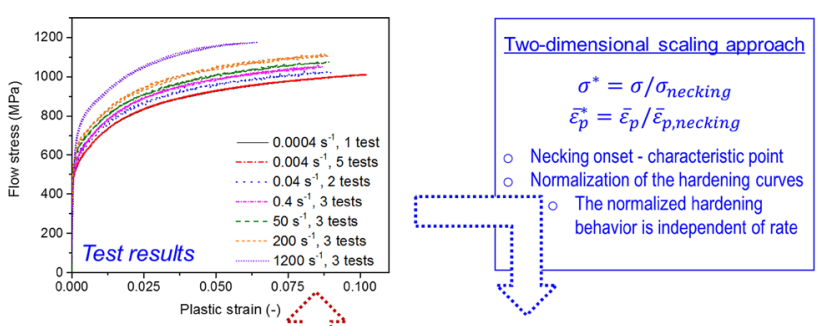

$\sigma\left(\bar{\varepsilon}_{p}, \dot{\bar{\varepsilon}}\right)$

$=\sigma^{*}\left[\bar{\varepsilon}_{p} / \bar{\varepsilon}_{p, \text { necking }}\right] \cdot \sigma_{\text {necking }}$

- Normalized hardening curve $\sigma\left(\bar{\varepsilon}_{p}, \dot{\bar{\varepsilon}}_{p}\right)=\sigma^{*}\left(\bar{\varepsilon}_{p}^{*}\right)$

2 Rate-dependence of necking onset $\sigma_{\text {necking }}\left(\dot{\bar{\varepsilon}_{p}}\right)$ $\bar{\varepsilon}_{p, \text { necking }}\left(\dot{\bar{\varepsilon}}_{p}\right)$

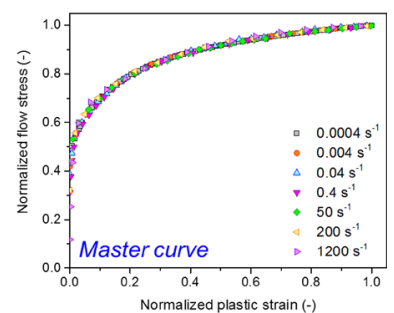

Keywords Rate-dependent material - Constitutive behavior $\cdot$ Dynamic testing $\cdot$ Necking behavior

\section{Introduction}

For the purpose of lightweight design, High Strength Steels (HSS) have been widely applied to auto-bodies. Compared with conventional steels, the HSS exhibits keep good formability while their strength is largely increased. This advantage makes them one of the promising materials for energy absorbing structures during automobile crash process. Therefore, in the past decade, HSS materials, especially Advanced High Strength Steels (AHSS), have achieved great success in the automotive industry. 
During stamping of the body part and crash events, the strain rate can reach $100 \mathrm{~s}^{-1}$ or more. Thus a practical material model must include the strain rate effect, which has been one of the most important issues in the characterization work. As investigated by previous researchers, most HSS materials are strain rate sensitive, including the HSLA-65 steel studied by Nemat-Nasser and Guo [1], the DP600 sheet and tube materials studied by Thompson [2], the DP600 sheet material studied by Yu et al. [3], the TRIP590 and DP780 sheet materials studied by Huh et al. [4] and DP800 studied by Khan et al. [5]. A comprehensive investigation on strain rate effect for a range of AHSS was reported by Yan and co-authors in a number of publications [6-9]. More recently, Kim et al. [10] investigated the dynamic mechanical behavior of a DP780 sheet, a DP980 sheet and a TRIP780 sheet. Their test results showed that the strength of the two studied DP steels is positively strain rate dependent while the case of the TRIP steel is much more complex because of the transformation kinetics.

To characterize the strain rate dependence, a number of rate-dependent material models have been proposed, known as visco-plasticity models. Existing visco-plasticity models can be generally classified into two types [11, 12], i.e. physics-based and phenomenological. The former usually involves micro-mechanism concepts. For instance, the widely-used Zerilli-Armstrong model [13], which is based on dislocation mechanics, takes the crystal structure of materials into consideration and introduces two different constitutive functions for BCC and FCC materials respectively.

The latter type, phenomenological model, is commonly purely empirical. Thanks to the calibrating convenience and computational efficiency, they are especially popular for industrial use. Johnson and Cook [14] proposed one of the most frequently-used phenomenological models, which multiplicatively decomposes the overall flow stress into three separate functions, strain hardening, strain rate effect and temperature dependence. These three effects are uncoupled in the Johnson-Cook model.

In this multiplicative decomposing approach of the Johnson-Cook model, the dynamic response can be considered as a scaled result of the quasi-static response. If plotted in the coordinate system of flow stress versus plastic strain, the dynamic curve under a certain strain rate is obtained by scaling the quasi-static curve along the flow stress axis by multiplying the strain rate effect function value and temperature dependence function value. This one-dimensional scaling approach is relatively simple and convenient to be coded into commercial Finite Element (FE) software. Therefore, it has been widely accepted by a great number of researchers $[2,12$, 15-18].
Despite its popularity and success, there are also some studies questioning the accuracy of the Johnson-Cook model, especially its strategy of uncoupling the effects of strain and strain rate. Liang and Khan [19] pointed out that the JC model is 'work-hardening rate increasing', which means its work-hardening rate becomes greater as the strain rate increases. This shortcoming makes it unable to characterize the 'work-hardening rate decreasing' materials, such as the tantalum they studied. To solve this problem, Khan and Liang [20] concluded that strain and strain rate must have some coupled effects on the description of work-hardening relation of the materials. Therefore, the Khan-Huang-Liang model, which was proposed early in 1992 [21], was utilized and developed in the later studies by the same research group [22-24]. Moreover, in one of their recent publications [11], the Khan-Liu model was proposed by coupling the effects of strain, strain rate and temperature all together.

Meanwhile, great progresses have also been achieved in the field of experimental mechanics. Traditional test methods, including the universal test machine for tests below $1 \mathrm{~s}^{-1}$ and the Hopkinson bar generally for tests above $500 \mathrm{~s}^{-1}$ [25-30], are relatively limited for comprehensively calibrating a rate-dependent model. The gap between these two categories, known as the intermediate strain rate range was seldom investigated in the past. However, thanks to the improvement in testing and measuring techniques in the last decade, such as the hydraulic power driving test machine and drop tower [17, 31-34], a wider strain rate range has been accessible for studying.

The intermediate range is of great importance to automotive industry because the maximum local strain rate is around $500 \mathrm{~s}^{-1}$ during vehicle accidents [17]. Most of the existing visco-plasticity models were proposed and developed based on experimental data under quasi-static and high strain rate loading conditions. Therefore, researchers are curious about the performance of these models in the intermediate strain rate range. In this area, great efforts have been devoted by Huh and co-authors [4, 17, 35] to characterize the mechanical behaviors of materials in a large range of strain rate, especially the intermediate range. Some improvements in the experimental methods were reported by Thompson [2], Larour et al. [36] and Gruben et al. [37]. But to the best knowledge of the authors, existing test data and modeling work for the intermediate strain rate range are still insufficient for wide industrial use.

The main purpose of this paper is to characterize the rate-dependent mechanical behavior of a DP780 steel sheet. The whole study starts from the uniaxial tension tests under a broad range of strain rates, which is exhibited in "Experiments" section. "Experimental Results and Analysis" section presents the test results and an in-depth analysis of the strain rate dependence. Constitutive 
modeling is developed in "Constitutive Modeling" section, and a new phenomenological model is proposed based on a two-dimensional scaling processing. Discussions of the calibration method and the applicability of the model are presented in "Discussions About the Application of the New Model" section.

\section{Experiments}

\section{Materials and Specimens}

The studied material in the present paper is a DP780 steel (AHSS) sheet with a thickness of $1.4 \mathrm{~mm}$. This grade is usually used for energy absorbing components in vehicles. More details such as chemical composition of the studied materials can be found in the previous work by the authors $[38,39]$.

A series of uniaxial tension tests are carried out, covering a strain rate range from $10^{-4}$ to $10^{3} \mathrm{~s}^{-1}$. To achieve the target strain rates, different sets of loading speed and specimen gage length are designed (see Table 1). The dimensions of the specimens with different gage length are shown in Fig. 1. Previous work by the authors has shown that the difference in gage length will result in a deviation of the post-necking behavior, but have no effect on the prenecking behavior of metal materials [33]. Therefore, the present study will be based on the analysis of the tensile behavior before the onset of necking.

\section{A Multiple-Machine System for Tests from Quasi- Static to Dynamic}

To get different loading speeds, a multiple-machine system consisting of a Universal Test Machine (UTM), an Intermediate Strain-rate Test Machine (ISTM) and a SplitTensile-Hopkinson Bar (STHB) is adopted. The UTM (CRIMS DDL50) is suitable for tests under the strain rate up to $1 \mathrm{~s}^{-1}$, while the STHB can cover the range above
$500 \mathrm{~s}^{-1}$. For the intermediate range, the custom made ISTM is used, shown in Fig. 2. The machine is driven by hydraulic power, and has a maximum loading speed of $2 \mathrm{~m} \mathrm{~s}^{-1}$.

Such a multiple-machine system is very common in studying the strain rate effect on material properties. However, the prerequisite issue-the compatibility between different machines-is seldom investigated. Therefore, the authors carried out an experimental study, and the reliability of the test machine system was verified by the test results [33]. The dynamic tests at the highest strain rate $\left(1200 \mathrm{~s}^{-1}\right)$ were carried out by another research group using a STHB, the schematic of which is shown in Fig. 3. This device is not outside the regular application of the one-dimensional wave theory, except that the loading impulse is generated by the tension-to-fracture process of an aluminum bar. By changing the geometry of the aluminum bar, the loading input could be easily adjusted, and such a design could effectively reduce the system ringing because the impulse is much smoother than direct impact between metal hammers. More details about the STHB and their testing method could be found in earlier publications $[15,40]$. All the tests are performed at room temperature (around $20{ }^{\circ} \mathrm{C}$ ).

\section{Reducing the System Ringing for Tests Under Intermediate Strain Rates}

Data oscillation caused by system resonance is one of the most significant and commonly-encountered problem for the tests under intermediate strain rates [34]. Load input methods, specimen geometry, clamping methods and measurement devices are the four well-known factors which are closely related to this ringing effect. Each of them are carefully designed for the dynamic tests of this present study, such as choosing suitable rubber layers to avoid direct impacts in the loading system and eliminating the gaps between the specimen and grips.

Table 1 Experiment matrix for seven different strain rate test groups

\begin{tabular}{lllll}
\hline Target strain rate $\left(\mathrm{s}^{-1}\right)$ & Specimen type & Loading speed $\left(\mathrm{m} \mathrm{s}^{-1}\right)$ & Test machine & True plastic strain rate $\left(\mathrm{s}^{-1}\right)$ \\
\hline 0.0004 & 1 & $8.33 \times 10^{-6}(0.5)^{\mathrm{a}}$ & UTM & 0.00036 \\
0.004 & 1 & $8.33 \times 10^{-5}(5)$ & UTM & 0.0033 \\
0.04 & 1 & $8.33 \times 10^{-4}(50)$ & UTM & 0.035 \\
0.4 & 1 & $8.33 \times 10^{-3}(500)$ & UTM & 0.223 \\
50 & 1 & $1.00 \times 10^{0}$ & ISTM & 12 \\
200 & 2 & $2.00 \times 10^{0}$ & ISTM & 93 \\
1200 & 3 & - & STHB & $-{ }^{\mathrm{b}}$ \\
\hline
\end{tabular}

${ }^{\mathrm{a}}$ Speeds in parentheses are in the unit of $\left(\mathrm{mm} \mathrm{min}^{-1}\right)$

b DIC is not used for STHB tests, true plastic strain is not calculated 
Moreover, based on the investigations by the authors, reducing the mass of the measuring part of the system, between the load applying point and the fixed end of the sensor, can greatly reduce the data oscillation. Therefore, a lightweight customized load sensor is adopted at the static end of the machine, shown in Figs. 2a and 4. During the test, one end of the specimen is directly clamped by the movable grip which is driven by hydraulic actuator, and the other end is fixed to Part I of the customized sensor. Strain gauges are attached to Part II of the sensor to capture the signal of load. Part III is clamped sturdily by the fixed grip of the machine. A previous study by the authors has shown that the sensor is very effective in reducing the data oscillation compared with commercial load sensors [32].

\section{Deformation Measurement}

In the tests of the UTM and ISTM, deformation measurements were accomplished by the 2D Digital Image
Fig. 1 Specimen for tension tests under different strain rates (unit: $\mathrm{mm}$ )

Fig. 2 a Schematic and b photograph of the measurement chain for intermediate strain rate loading

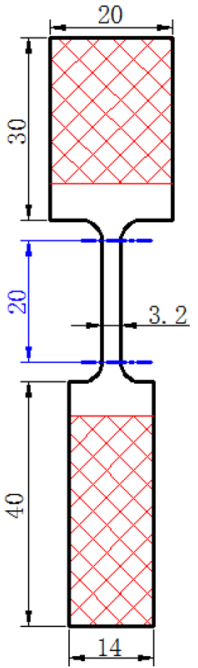

Type-1

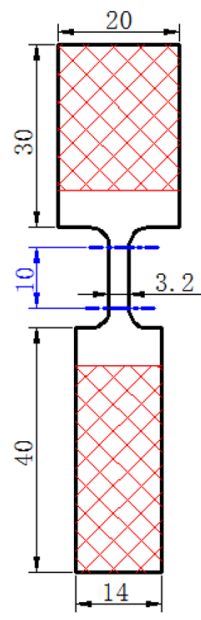

Type-2

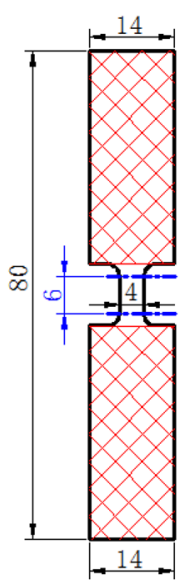

Type-3 a

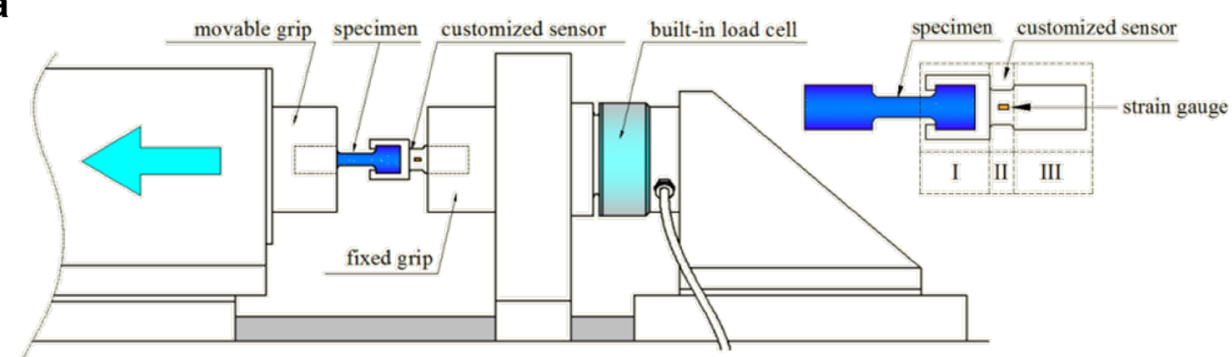

b

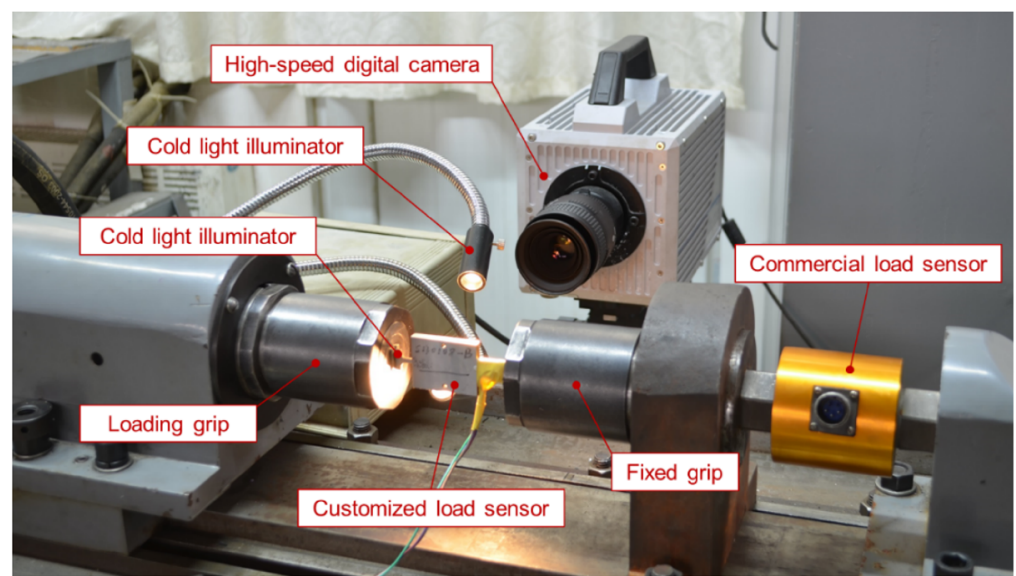


Fig. 3 Schematic of the SplitTensile-Hopkinson Bar [15]

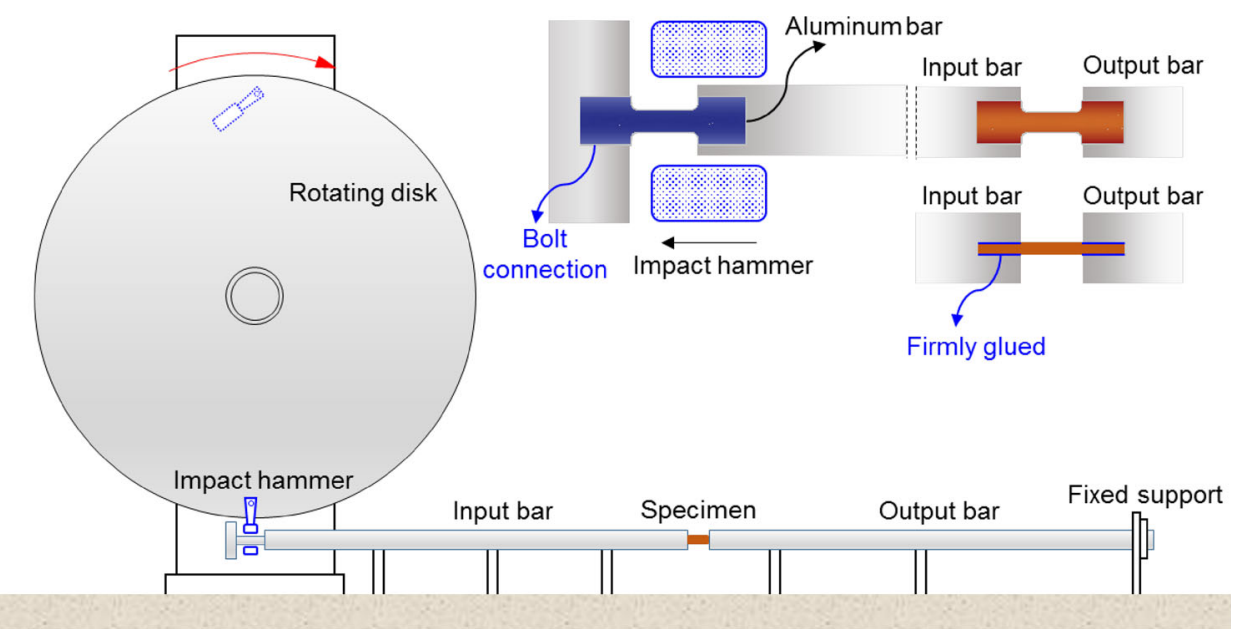

a

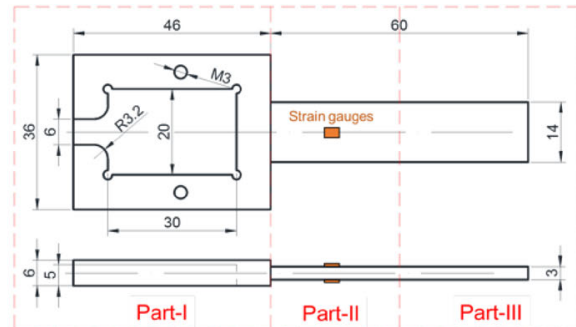

b

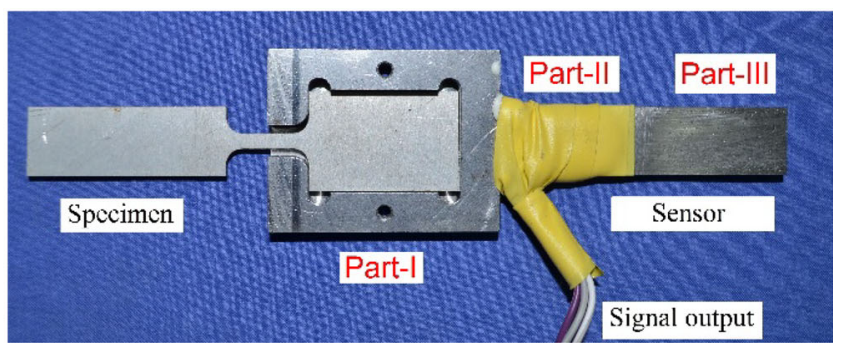

Fig. 4 Customized load sensor to reduce the system ringing during dynamic tests

Correlation (DIC) system (VIC-2D). For quasi-static tests, an industrial CCD camera (DH-HV1351) is sufficient, while for dynamic tests, a high-speed digital camera (Photron 675K) was used to record enough data points during the transient test processes (see Fig. 2b). For each single test, at least 250 images are captured during the plastic deformation stage. In the STHB tests, the strain of the material is measured by strain gauges.

\section{Experimental Results and Analysis}

\section{Experimental Results}

True stress-strain curve is processed based on the nominal stress-strain curve obtained from the tests.

$\left\{\begin{array}{l}\sigma_{\text {true }}=\sigma_{\text {nominal }} \cdot\left(1+\varepsilon_{\text {nominal }}\right) \\ \varepsilon_{\text {true }}=\ln \left(1+\varepsilon_{\text {nominal }}\right)\end{array}\right.$

This study will be focused on pre-necking hardening behavior. The onset of necking of each test is determined by Swift's theory [41].

$\sigma_{\text {true }}=d \sigma_{\text {true }} / d \varepsilon_{\text {true }}$

Pre-necking hardening curves of the material are exhibited in Fig. 5. For dynamic test, at least three repeats

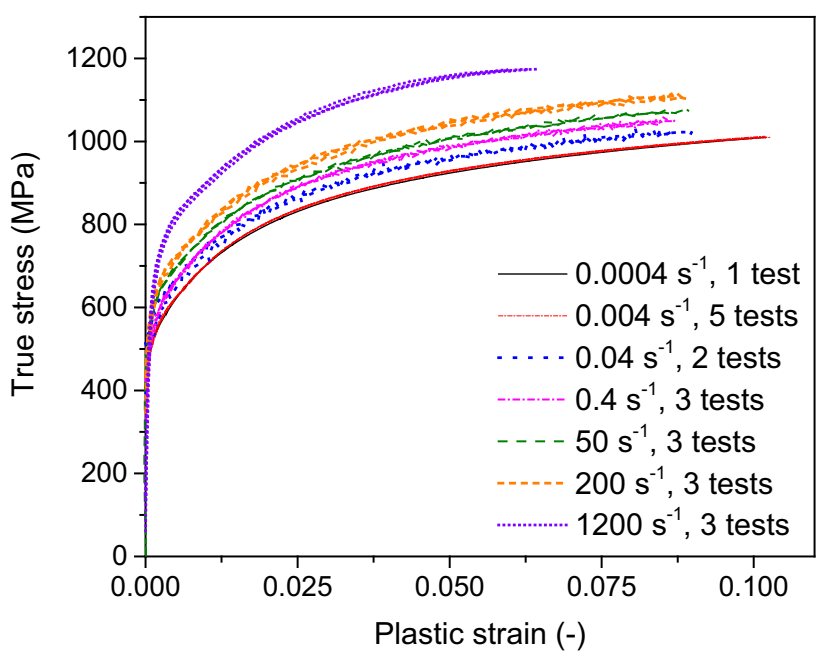

Fig. 5 Hardening curves under different strain rates for the DP780 sheet

of tests are guaranteed, and the repeatability of the test was very good (curves under the same strain rate can almost superimpose upon each other). No data oscillation is observed.

From the test results, it can be clearly observed that the material displays a positive strain rate effect in stress, i.e. 
the stress increases with the rise in strain rate. How to quantify this strain rate effect is the next task of the present paper.

\section{True Plastic Strain Rate During Tests}

The strain rates $\dot{\varepsilon}$ in the first column of Table 1, called target strain rates, are calculated by dividing loading speed with gage length. In a practical test, the loading speed may be a little lower than the target one because there is always an accelerating period to reach it. Moreover, the plastic strain rate $\dot{\bar{\varepsilon}}_{p}$ is preferable when modeling the strain rate effect rather than the strain rate $\dot{\varepsilon}$. For this reason, the true plastic strain rate for each of the tests is calculated as the average plastic strain rate from yield point (time $t_{\text {yield }}$ ) to the onset of necking (time $t_{\text {necking }}$ ).

$\dot{\bar{\varepsilon}}_{p}=\bar{\varepsilon}_{p, \text { necking }} /\left(t_{\text {necking }}-t_{\text {yield }}\right)$

This average value is valid as long as the oscillation in strain rate is small. According to the ISO standard [42], the maximum deviation from the average value should be less than $30 \%$. With the experimental techniques introduced in "Experiments" section, the results of the present study can meet this requirement satisfactorily [33]. The true plastic strain rates for each of the groups are listed in Table 1. In fact, the difference between plastic strain rate and strain rate is relatively small, and many publications do not distinguish between them. In this study, the true plastic strain rate will be used for modeling and fitting. But for convenience, it will still be referred to as 'strain rate'.

\section{Effect of Thermal Softening}

Though all the tests are carried out at room temperature, the effect of thermal softening still exists because of the temperature rise that comes from the plastic deformation. Under quasi-static conditions, this effect can be neglected since there is plenty of time for thermal conduction. However, under high-speed dynamic conditions, the deformation time period is so short that the material undergoes an adiabatic procedure. According to existing publications $[12,43]$, the temperature rise can be determined as

$$
\Delta T=\frac{\eta}{\rho C_{p}} \int \sigma d \bar{\varepsilon}_{p}
$$

where $\eta$ is the fraction of heat conversion from plastic deformation, $\rho$ is the density of the studied material, and $C_{p}$ is the heat capacity at constant pressure. Steels usually have a density of $7850 \mathrm{~kg} \mathrm{~m}^{-3}$ and a heat capacity of $420 \mathrm{~J} \mathrm{~kg}^{-1} \mathrm{~K}^{-1}$. For most materials in adiabatic situation, the fraction of heat conversion could be regarded as 0.9 .
Therefore, for the studied material, the temperature rise is about $22{ }^{\circ} \mathrm{C}$, calculated from the pre-necking hardening curves. As suggested by the Johnson-Cook model, the thermal effect on stress can be estimated as

$h(T)=1-\left[\left(1-T_{r}\right) /\left(T_{m}-T_{r}\right)\right]^{m}$

where $T_{r}$ and $T_{m}$ are the reference temperature (usually set as room temperature, $293 \mathrm{~K}$ ) and melting temperature (approximately $1700 \mathrm{~K}$ for steels). And according to a recent work on similar High Strength Steel materials [12], the coefficient $m$ in Eq. (5) is about 0.92 .

Letting $T$ equal to $315 \mathrm{~K}\left(42{ }^{\circ} \mathrm{C}\right)$, the value of $h$ in Eq. (5) turns out to be 0.98 , very close to 1.00 (no influence). Therefore, in the current study, the thermal softening effect is neglected. This is consistent with the statement by Roth and Mohr [12] that thermal effect is negligible for pre-necking behavior.

\section{Constitutive Modeling}

\section{One-Dimensional Scaling}

Existing models, such as the Johnson-Cook model introduced in "Introduction" section, commonly adopts a one-dimensional scaling strategy, which approximate dynamic stress-strain curve by scaling up the quasi-static hardening curve by a function of strain rate. Cowper and Symonds [44] proposed a power law for this strain rate dependence function, which has gained lots of popularity in industry and is usually combined with the Johnson-Cook model in its decomposition-form expression.

$\sigma\left(\dot{\bar{\varepsilon}}_{p}, \overline{\bar{\varepsilon}}_{p}\right)=\sigma_{\text {static }}\left(\overline{\bar{\varepsilon}}_{p}\right) g\left(\dot{\bar{\varepsilon}}_{p}\right)=\sigma_{\text {static }}\left[1+\left(\dot{\bar{\varepsilon}}_{p} / D_{1}\right)^{D_{2}}\right]$

where $\sigma_{\text {static }}$ is usually understood by the quasistatic hardening curve corresponding to the strain rate of around $10^{-4} \mathrm{~s}^{-1}$. The above simple equation has become one of the most cited results in the area of dynamic plasticity. Using the experimental data of Fig. 5 and choosing as a reference quasi-static loading the curve corresponding to $\dot{\bar{\varepsilon}}_{p}=0.0004 \mathrm{~s}^{-1}$, the plot of the normalized stress $\sigma^{*}$, defined by

$\sigma^{*}=\sigma\left(\dot{\bar{\varepsilon}}_{p}, \bar{\varepsilon}_{p}\right) / \sigma_{\text {static }}$

is shown in Fig. 6. With the above normalization, the curves corresponding to different strain rates fall within a rather narrow band. In many engineering applications this description is considered to be sufficiently accurate. A new, two-dimensional normalization is proposed in the present paper which brings the test results of all strain rates into a single, master curve. This feature, along with the applications of the new dynamic test methodology constitutes the main contribution of the present paper. 


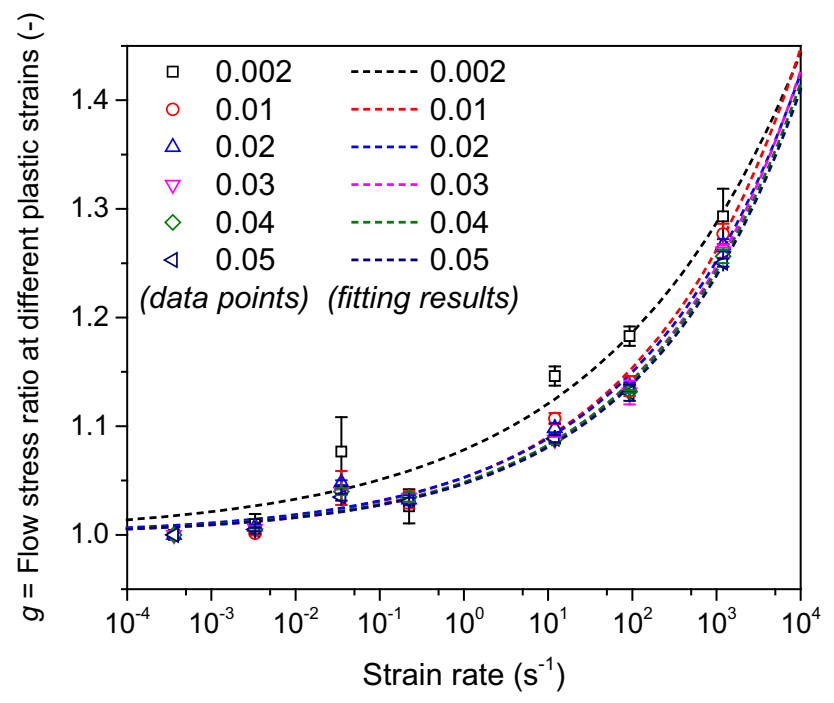

Fig. 6 Strain rate effect in stress for the DP780 at different plastic strains (dashed curves fitting results using Cowper-Symonds function)

\section{Two-Dimensional Scaling Approach}

According to the test data, a two-dimensional scaling (2Dscaling) approach is applied in further analysis of the test results. In this approach, the onset of necking is utilized as a 'characteristic point' to normalize the hardening curve. The normalized plastic strain is defined as

$\left\{\begin{array}{l}\sigma^{*}=\sigma / \sigma_{\text {neck }} \\ \bar{\varepsilon}_{p}^{*}=\bar{\varepsilon}_{p} / \bar{\varepsilon}_{p, \text { neck }}\end{array}\right.$

where the superscript "*" denotes the normalized term, and the subscript "neck" means the value at the onset of necking.

The co-ordinate of the necking point of any stress-strain curve is calculated from the Swift stability condition for uniaxial tension, given by Eq. (2). Using a simple MATLAB program, the values for each curve are obtained and listed in Table 2 and shown graphically in Fig. 8.

The normalized hardening curves, defined by Eq. (8), are shown in Fig. 7. A master curve is observed that all the

Table 2 Plastic strain and flow stress of the necking point

\begin{tabular}{lll}
\hline Target strain rate $\left(\mathrm{s}^{-1}\right)$ & Plastic strain $(-)$ & Flow stress $(\mathrm{MPa})$ \\
\hline 0.0004 & $0.106 \pm 0.001$ & $1013.70 \pm 1.20$ \\
0.004 & $0.104 \pm 0.002$ & $1013.42 \pm 1.74$ \\
0.04 & $0.092 \pm 0.002$ & $1029.30 \pm 4.02$ \\
0.4 & $0.094 \pm 0.005$ & $1061.60 \pm 14.24$ \\
50 & $0.086 \pm 0.002$ & $1077.32 \pm 6.56$ \\
200 & $0.087 \pm 0.006$ & $1130.37 \pm 9.46$ \\
1200 & $0.062 \pm 0.004$ & $1171.05 \pm 8.38$ \\
\hline
\end{tabular}

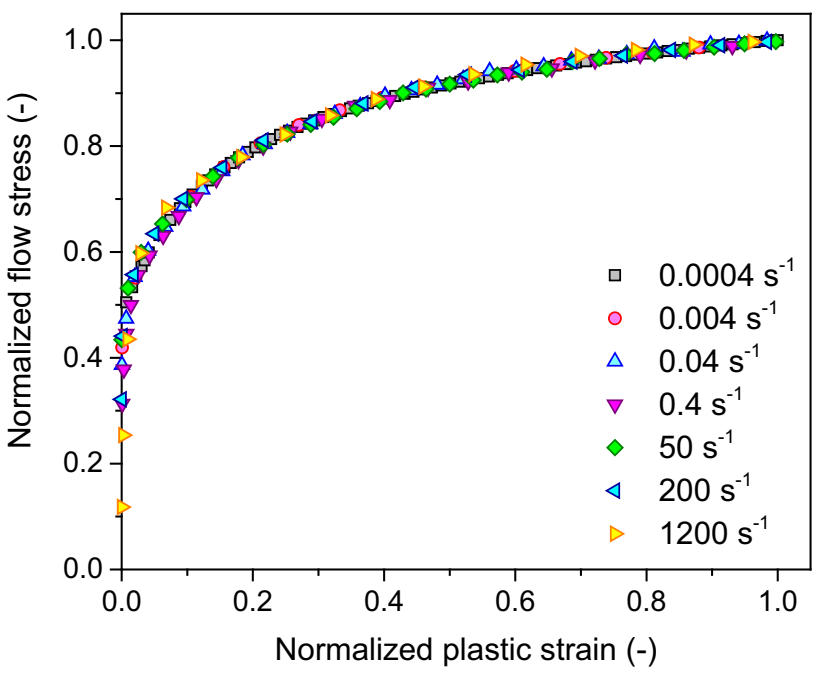

Fig. 7 Normalized hardening curves for the studied DP780

normalized hardening curves superimpose upon each other for the DP780 steel, which means the normalized hardening behavior is independent of strain rate in the pre-necking range.

\section{A New Model Based on a Master Curve}

Such a master curve, independent of strain rate, is of special importance for constitutive modeling. Based on this curve, hardening curve at an arbitrary strain rate can be obtained by multiplying the $\bar{\varepsilon}_{p}^{*}$ and $\sigma^{*}$ with the plastic strain and flow stress at the onset of necking of that strain rate, respectively. Therefore, a new model can be developed, consisting of two separate parts:

(1) A master curve to describe the normalized hardening behavior $\sigma^{*}\left(\bar{\varepsilon}_{p}^{*}\right)$;

(2) Rate-dependence of the onset of necking, including the plastic strain $\bar{\varepsilon}_{p, \text { neck }}\left(\dot{\bar{\varepsilon}}_{p}\right)$ and the flow stress $\sigma_{\text {neck }}\left(\dot{\bar{\varepsilon}}_{p}\right)$. Both of them are functions of strain rate.

Substituting these terms into Eq. (8), the 2D-scaling model can be expressed as

$\sigma\left(\bar{\varepsilon}_{p}, \dot{\bar{\varepsilon}}_{p}\right)=\sigma^{*}\left[\bar{\varepsilon}_{p} / \bar{\varepsilon}_{p, \text { neck }}\left(\dot{\bar{\varepsilon}}_{p}\right)\right] \cdot \sigma_{\text {neck }}\left(\dot{\bar{\varepsilon}_{p}}\right)$

The master curve can be fitted by either Ludwik or Voce law, or their combination

$$
\begin{aligned}
& \sigma^{*}\left(\bar{\varepsilon}_{p}^{*}\right)=C_{5} \underbrace{\left[C_{1}+\left(1-C_{1}\right)\left(\bar{\varepsilon}_{p}^{*}\right)^{C_{2}}\right]}_{\text {Ludwik }}+ \\
& \left(1-C_{5}\right) \underbrace{\left[C_{3}\left(1-\exp \left(C_{4}\left(\vec{\varepsilon}_{p}^{*}-1\right)\right)\right)+1\right]}_{\text {Voce }}
\end{aligned}
$$


where $C_{5}$ is the weighting factor of the Ludwik and Voce law.

It is observed that the necking stress and strain can be approximated by the following functions.

$$
\begin{aligned}
& \sigma_{\text {neck }}\left(\dot{\bar{\varepsilon}}_{p}\right)=\sigma_{\text {static,neck }}\left[1+\left(\dot{\bar{\varepsilon}}_{p} / D_{1}\right)^{D_{2}}\right] \\
& \bar{\varepsilon}_{p, \text { neck }}\left(\dot{\bar{\varepsilon}}_{p}\right)=\bar{\varepsilon}_{p, \text { static,neck }}\left[1-\left(\dot{\bar{\varepsilon}}_{p} / D_{3}\right)^{D_{4}}\right]
\end{aligned}
$$

where the subscript "static" denotes the necking point at the lowest strain rate, $4 \times 10^{-4} \mathrm{~s}^{-1}$.

The fitting results are plotted in Fig. 8. Functions like Cowper-Symonds form can accurately describe the ratedependence of necking stress, but have a relatively limited ability for the plastic strain. This is because the plastic strain of the onset of necking varies with the strain rate in a very complex trend, even not monotonically in the intermediate range. This observed trend agrees well with the studies by other researchers [2, 45].

By substituting the rate dependence functions of the necking point (Eqs. 11 and 12) into the master curve (Eq. 10), the 2D-scaling model can be expressed explicitly. For example, when the weighting factor $C_{5}=1$, a simplified expression is

$$
\begin{aligned}
\sigma\left(\overline{\bar{\varepsilon}}_{p}, \dot{\bar{\varepsilon}}_{p}\right)= & {\left[C_{1}+\left(1-C_{1}\right)\left(\frac{\overline{\bar{\varepsilon}}_{p}}{\overline{\bar{\varepsilon}}_{p, \text { static, neck }}\left[1-\left(\dot{\bar{\varepsilon}}_{p} / D_{3}\right)^{D_{4}}\right]}\right)^{C_{2}}\right] } \\
& \cdot\left\{\sigma_{p, \text { static neck }}\left[1+\left(\dot{\bar{\varepsilon}}_{p} / D_{1}\right)^{D_{2}}\right]\right\}
\end{aligned}
$$

For the present DP780, the combined form of Ludwik and Voce is used. Figure 9a exhibits the characterization results by the $2 \mathrm{D}$-scaling model, which is of very satisfactory accuracy.

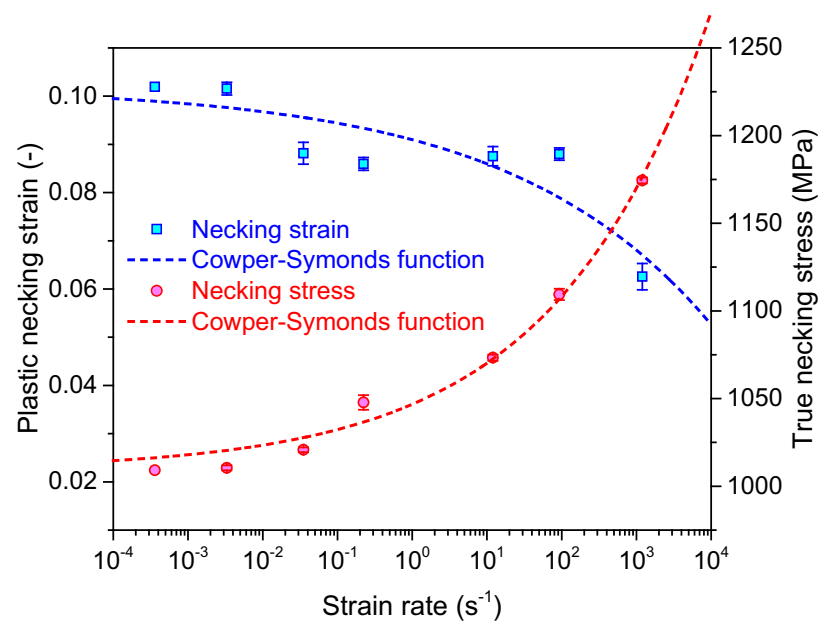

Fig. 8 Fitting of the plastic strain and true stress at the onset of necking

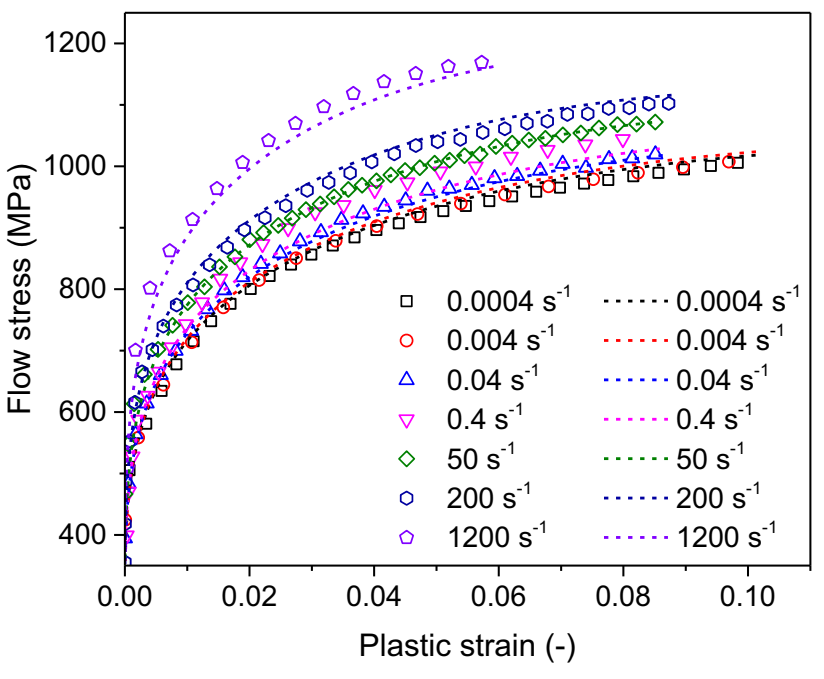

Fig. 9 Characterization results by the 2D-scaling model (points test data; dashed lines characterization results by the model)

\section{Discussions About the Application of the New Model}

\section{Calibration Method}

The main merit of the new model is that it provides a practical method for determining strain rate properties of metallic materials. The present simple and straightforward calibration procedure involves only one quasi-static and two dynamic uniaxial tension test (for the lowest cost). The quasi-static test should be performed at strain rate at the order of $10^{-3} \mathrm{~s}^{-1}$. The user should determine the magnitude of strain rate in the dynamic test, but to have sufficient accuracy, those strain rate should differ at least by an order of magnitude. For example, for the present studied material, taken as the quasi-static and low-speed dynamic test at $\dot{\bar{\varepsilon}}=0.4 \mathrm{~s}^{-1}$. Both these tests were obtained with the Universal Test Machine. The third test at $\dot{\overline{\varepsilon_{p}}}=200 \mathrm{~s}^{-1}$ was obtained by means of the customized Intermediate Strainrate Test Machine. It is here that the advantage of a present experimental technique for intermediate strain rates will be most useful.

The calibration will include the following six steps:

(1) Convert the measured load-displacement relations for the prescribed strain rate to the engineering stress-strain curve and then to the true stress-strain curve. There is no need to develop an analytical fit of the measured curves.

(2) Use the Swift criterion to obtain the necking points for all the three tests (one static and two high strain rate). This will provide the magnitude of the necking strain $\bar{\varepsilon}_{p, n e c k}$ and the corresponding necking stress $\sigma_{\text {neck }}$. As mentioned before, this could be done using 
Table 3 Calibration coefficients of the new model for the studied DP780

\begin{tabular}{llllllllll}
\hline Coefficient & $C_{1}(-)$ & $C_{2}(-)$ & $C_{3}(-)$ & $C_{4}(-)$ & $C_{5}(-)$ & $D_{1}\left(\mathrm{~s}^{-1}\right)$ & $D_{2}(-)$ & $D_{3}\left(\mathrm{~s}^{-1}\right)$ & $D_{4}(-)$ \\
\hline Value & -0.676 & 0.274 & 1.406 & 0.260 & 0.548 & $6.126 \times 10^{6}$ & 0.211 & $8.562 \times 10^{5}$ & 0.163 \\
\hline
\end{tabular}

a simple MATLAB code, and this method is suggested here. Alternatively, one can fit the measured true stress-strain curve by a smooth analytical function and then use Eq. (2). It is recommended to find coordinate of the necking point numerically.

(3) The next step is to find the coefficient of the strain rate effect functions of the necking point for stress from Eq. (11). There are two unknowns in Eq. (11), $D_{1}$ and $D_{2}$. They are determined from the two dynamic tests, run respectively at 0.4 and $200 \mathrm{~s}^{-1}$. Then the unknown coefficients, $D_{1}$ and $D_{2}$, are found from the solution of the two linear algebraic equations.

(4) A similar procedure is repeated for the unknown coefficients $D_{3}$ and $D_{4}$ in Eq. (12) for the necking point of strain.

(5) Construct the master curve by normalizing the measured stress-strain curves for each of the above three strain rates. If done properly, the curve shown in Fig. 7 should be obtained. This normalized experimental curve should be fitted by the analytical function of Eq. (10). The unknown Coefficients $C_{1-5}$ can be determined by means of MATLAB minimization function fminsearch. Note that $\sigma^{*}$ should always be a positive value. Therefore, it is highly suggested that the users adopt $\sigma^{*}(0)=$ $\sigma_{\text {static,yeild }} / \sigma_{\text {static,neck }}$ as a boundary condition for this optimization process. The numerical values for all coefficients $C_{1-5}$ and $D_{1-4}$ for the studied DP780 are given in Table 3.

(6) With all the strain hardening and strain rate effect parameters known, the dynamic stress-strain curves for any chosen strain rate can be obtained by Eq. (13).

\section{Verification of the New Model}

A strain rate jump test [20] is carefully carried out with the multiple-machine test system. The specimen undergoes a series of loading speed changes in one test. The changes in the quasi-static zone (below $10^{-1} \mathrm{~s}^{-1}$ ) are realized by setting a piecewise velocity curve for loading in the universal test machine. For the change from 0.00034 to $2.67 \mathrm{~s}^{-1}$, the specimen is unloaded after the loadings in the UTM, and then moved to the intermediate strain rate machine for dynamic test.

Given the test data of $\bar{\varepsilon}_{p}$ at any moment, the strain rate $\dot{\bar{\varepsilon}}_{p}(t)$ can be obtained by taking derivation of the strain- time curve, and thus the corresponding stress $\sigma(t)$ can be predicted by substituting the strain and strain rate into the 2D-scaling model. As shown in Fig. 10, experimental results and model prediction are in good agreement with each other, validating the $2 \mathrm{D}$-scaling model and the calibrated coefficients.

\section{Applicability of the 2D-Scaling Model}

Four more DP steels are investigated, of which, one is tested by the authors and the other three are extracted from the open literature. The normalized hardening curves in Fig. 11 agree quite well with the previous results-almost all the DP steels have a rate-independent master curve, indicating that the 2D-scaling method can be applied to these DP steels with good accuracy.

As far as the authors are concerned, even if there is a deviation between the normalized hardening curves in some cases (see Fig. 11c), the 2D-scaling model still has advantage over the conventional 1D-scaling models because it can largely increase the accuracy of the prediction of the necking point, which presents the peak load of the material and has special importance in industrial use.

What is more interesting to the users of the model perhaps is its applicability to other materials, such as other steels, aluminum alloys and non-metal materials. More experimental and analytical work is needed to draw a complete conclusion, and therefore the authors are currently working on the two-dimensional normalization of High Strength Low Alloy steels as a next step.

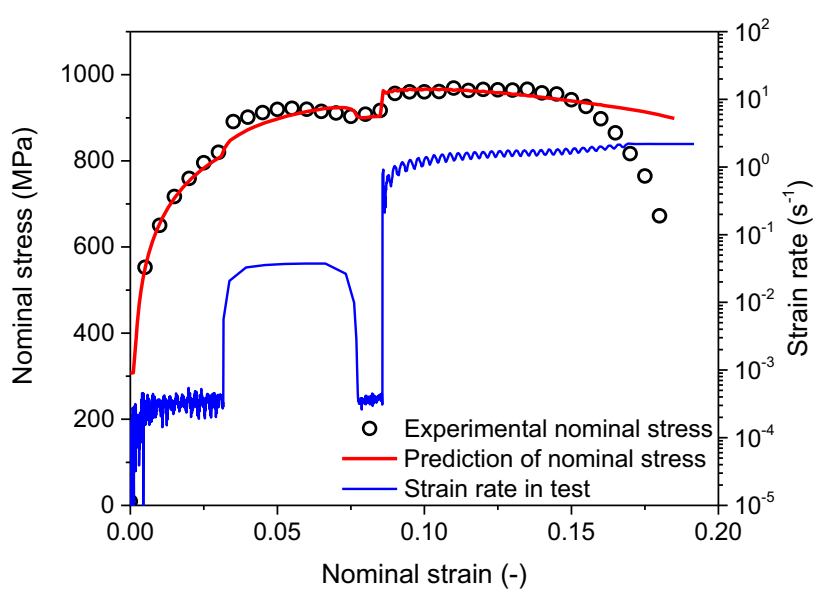

Fig. 10 Strain rate jump test result and model prediction in terms of nominal curve 

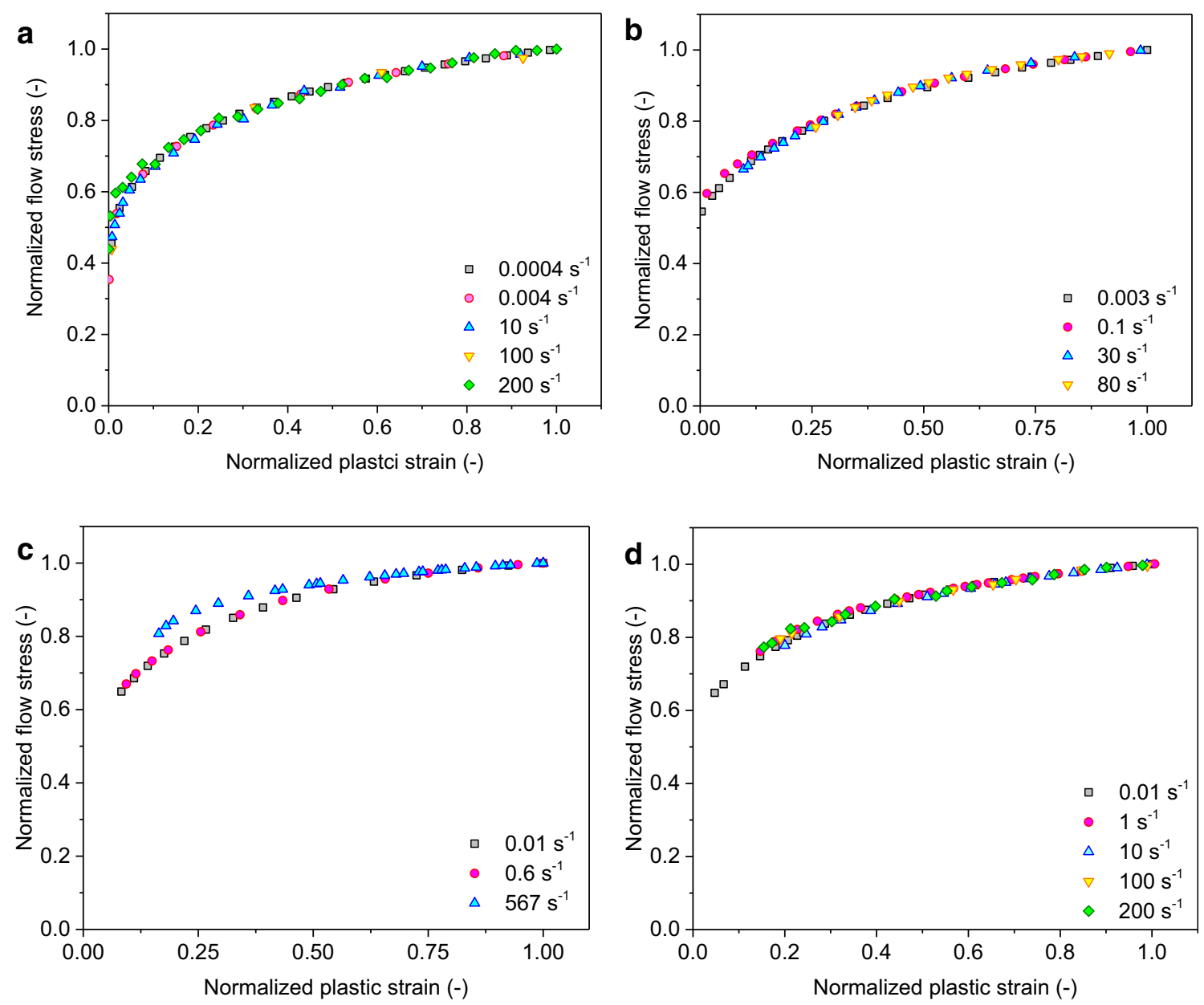

Fig. 11 Normalized hardening curves of a the DP600 tested by the authors b the DP600 tested by Thompson [2] $\mathbf{c}$ the DP590 tested by Roth and Mohr [12] and $\mathbf{d}$ the DP780 tested by Kim et al. [10]

\section{Conclusions}

In this paper, tension tests under seven strain rates are carried out for a DP780 steel with a multiple-machine system consisting of a universal test machine, a hydraulic machine and a split Hopkinson bar. Test results show that the material has a positive strain rate dependence in flow stress and a generally negative strain rate dependence in necking strain.

A new data analyzing approach, 2D-scaling, is proposed, which uses the onset of necking as a characteristic point to normalize the hardening curve under each strain rate. A master curve is observed for the DP780 steel, i.e. the normalized hardening behavior is rate-independent. Based on this master curve, a new rate-dependent model is proposed. The accuracy of the model is validated by a strain rate jump test, and the applicability of the model turns out to be very good for most DP steels.

Acknowledgments This work is supported by the National Program of International S\&T Cooperation under the project number of
2014DFG71590. The authors would like to express their thanks to Mr. Lingchen Ma, Mr. Xinqi Wei for their great assistance with the experimental work, and to Dr. Gongyao Gu for his constructive suggestions. Thanks also to Prof. Yang Wang and Dr. Jun Zhang of University of Science and Technology of China for their help in the STHB tests.

\section{References}

1. Nemat-Nasser S, Guo W-G (2005) Thermomechanical response of HSLA-65 steel plates: experiments and modeling. Mech Mater 37(2):379-405

2. Thompson A (2006) High strain rate characterization of advanced high strength steels. Doctoral Thesis: University of Waterloo, Canada

3. Yu H, Guo Y, Lai X (2009) Rate-dependent behavior and constitutive model of DP600 steel at strain rate from $10^{-4}$ to $10^{3} \mathrm{~s}^{-1}$. Mater Des 30(7):2501-2505

4. Huh J, Huh H, Lee CS (2013) Effect of strain rate on plastic anisotropy of advanced high strength steel sheets. Int $\mathbf{J}$ Plast 44:23-46

5. Khan AS, Baig M, Choi S-H, Yang H-S, Sun X (2012) Quasistatic and dynamic responses of advanced high strength steels: experiments and modeling. Int J Plast 30:1-17 
6. Yan B, Xu K (2003) High strain rate behavior of advanced high strength steels for automotive applications. Iron Steelmak 30(6):33-42

7. Le Q, Kang HT, Kridli G, Khosrovaneh AK, Yan B (2009) Effect of prestrain paths on mechanical behavior of dual phase sheet steel. Int J Fatigue 31(4):607-615. doi:10.1016/j.ijfatigue.2008.03.028

8. Yan B, Urban D (2003) Characterization of fatigue and crash performance of new generation high strength steels for automotive applications. AIAI/DOE Technology Roadmap Program Report, Pittsburgh

9. Yan B, Belanger P, Citrin K (2001) Effect of forming strain on fatigue performance of a mild automotive steel. SAE Technical Paper

10. Kim J-H, Kim D, Han HN, Barlat F, Lee M-G (2013) Strain rate dependent tensile behavior of advanced high strength steels: experiment and constitutive modeling. Mater Sci Eng A 559:222-231

11. Khan AS, Liu HW (2012) Variable strain rate sensitivity in an aluminum alloy: response and constitutive modeling. Int J Plast 36:1-14

12. Roth CC, Mohr D (2014) Effect of strain rate on ductile fracture initiation in advanced high strength steel sheets: experiments and modeling. Int J Plast 56:19-44. doi:10.1016/j.ijplas.2014.01.003

13. Zerilli FJ, Armstrong RW (1987) Dislocation-mechanics-based constitutive relations for material dynamics calculations. J Appl Phys 61(5):1816-1825. doi:10.1063/1.338024

14. Johnson GR, Cook WH (1983) A constitutive model and data for metals subjected to large strains, high strain rates and high temperatures. In: Proceedings of the 7th International Symposium on Ballistics. The Hague, The Netherlands, pp 541-547

15. Wang Y, Zhou Y, Xia Y (2004) A constitutive description of tensile behavior for brass over a wide range of strain rates. Mater Sci Eng A 372(1):186-190

16. Smerd R, Winkler S, Salisbury C, Worswick M, Lloyd D, Finn M (2005) High strain rate tensile testing of automotive aluminum alloy sheet. Int J Impact Eng 32(1):541-560

17. Huh H, Lim J, Park S (2009) High speed tensile test of steel sheets for the stress-strain curve at the intermediate strain rate. Int J Automot Technol 10(2):195-204

18. Pack K, Roth CC (2016) The second Sandia Fracture Challenge: blind prediction of dynamic shear localization and full fracture characterization. Int J Fract. doi:10.1007/s10704-016-0091-0

19. Liang R, Khan AS (1999) A critical review of experimental results and constitutive models for BCC and FCC metals over a wide range of strain rates and temperatures. Int $\mathrm{J}$ Plast 15(9):963-980

20. Khan AS, Liang R (1999) Behaviors of three BCC metal over a wide range of strain rates and temperatures: experiments and modeling. Int J Plast 15(10):1089-1109

21. Khan AS, Huang S (1992) Experimental and theoretical study of mechanical behavior of 1100 aluminum in the strain rate range $10^{-5}-10^{4} \mathrm{~s}^{-1}$. Int J Plast 8(4):397-424. doi:10.1016/07496419(92)90057-J

22. Khan AS, Liang R (2000) Behaviors of three BCC metals during non-proportional multi-axial loadings: experiments and modeling. Int J Plast 16(12):1443-1458

23. Khan AS, Suh YS, Kazmi R (2004) Quasi-static and dynamic loading responses and constitutive modeling of titanium alloys. Int J Plast 20(12):2233-2248

24. Khan AS, Kazmi R, Farrokh B (2007) Multiaxial and non-proportional loading responses, anisotropy and modeling of Ti-6Al$4 \mathrm{~V}$ titanium alloy over wide ranges of strain rates and temperatures. Int J Plast 23(6):931-950

25. Hopkinson B (1914) A method of measuring the pressure produced in the detonation of high explosives or by the impact of bullets. Philos Trans R Soc Lond Ser A 213:437-456
26. Kolsky H (1949) An investigation of the mechanical properties of materials at very high rates of loading. Proc Phys Soc B 62(11):676-700

27. Mohr D, Gary G, Lundberg B (2010) Evaluation of stress-strain curve estimates in dynamic experiments. Int $\mathrm{J}$ Impact Eng 37(2):161-169

28. Chen WW, Song B (2010) Split Hopkinson (Kolsky) bar: design, testing and applications. Springer, Berlin

29. Rittel D, Ravichandran G (2005) High-strain-rate behavior of $\alpha$ iron under shear dominant loading conditions. Parameters 1:2

30. Tarigopula V, Hopperstad OS, Langseth M, Clausen AH, Hild F (2008) A study of localisation in dual-phase high-strength steels under dynamic loading using digital image correlation and $\mathrm{FE}$ analysis. Int J Solids Struct 45(2):601-619. doi:10.1016/j.ijsolstr. 2007.08.021

31. Bruce DM, Matlock DK, Speer JG, De AK Assessment of the strain-rate dependent tensile properties of automotive sheet steels. In: SAE 2004 World Congress and Exhibition, 2004

32. Xia Y, Zhu J, Wang K, Zhou Q (2016) Design and verification of a strain gauge based load sensor for medium-speed dynamic tests with a hydraulic test machine. Int J Impact Eng 88:139-152. doi:10.1016/j.ijimpeng.2015.10.004

33. Xia Y, Zhu J, Zhou Q (2015) Verification of a multiple-machine program for material testing from quasi-static to high strain-rate. Int J Impact Eng 86:284-294. doi:10.1016/j.ijimpeng.2015.07. 010

34. Xiao X (2008) Dynamic tensile testing of plastic materials. Polym Testing 27(2):164-178

35. Huh H, Ahn K, Lim JH, Kim HW, Park LJ (2014) Evaluation of dynamic hardening models for BCC, FCC, and HCP metals at a wide range of strain rates. $\mathrm{J}$ Mater Process Technol 214(7):1326-1340

36. Larour P, Bleck W, Raabe D (2010) Strain rate sensitivity of automotive sheet steels: influence of plastic strain, strain rate, temperature, microstructure, bake hardening and pre-strain. Doctoral Thesis: RWTH Aachen Aachen, Germany

37. Gruben G, Langseth M, Fagerholt E, Hopperstad OS (2016) Lowvelocity impact on high-strength steel sheets: an experimental and numerical study. Int J Impact Eng 88:153-171. doi:10.1016/j. ijimpeng.2015.10.001

38. Zhu J, Xia Y, Gu G, Zhou Q (2014) Extension of non-associated Hill48 Model for characterizing dynamic mechanical behavior of a typical high-strength steel sheet. In: ASME 2014 International Mechanical Engineering Congress and Exposition. American Society of Mechanical Engineers, pp V009T012A097V009T012A097

39. Zhu J, Xia Y, Luo H, Gu G, Zhou Q (2014) Influence of flow rule and calibration approach on plasticity characterization of DP780 steel sheets using Hill48 model. Int J Mech Sci 89:148-157

40. Cao K, Ma X, Zhang B, Wang Y, Wang Y (2010) Tensile behavior of polycarbonate over a wide range of strain rates. Mater Eng A 527(16-17):4056-4061

41. Swift HW (1952) Plastic instability under plane stress. J Mech Phys Solids 1(1):1-18

42. ISO (2011) Metallic materials-tensile testing at high strain rates-Part 2: Servo-hydraulic and other test systems

43. Sung JH, Kim JH, Wagoner R (2010) A plastic constitutive equation incorporating strain, strain-rate, and temperature. Int $\mathrm{J}$ Plast 26(12):1746-1771

44. Cowper GR, Symonds PS (1957) Strain-hardening and strain-rate effects in the impact loading of cantilever beams. DTIC Document

45. Ding L, Lin J, Min J, Pang Z, Ye Y (2013) Necking of Q\&P steel during uniaxial tensile test with the aid of DIC technique. Chin J Mech Eng 26(3):448-453 